\title{
Predicting survival after veno-arterial-ECMO first external validation of the SAVE-score
}

\author{
Mansour A., Roisne A., Nesseler N., Lavoué S., Seguin P., Flecher E. \\ CHU de Rennes, Dept of Anaesthesiology \& Intensive Care, Rennes, France
}

\section{Background and Goal of Study}

Veno-arterial-extracorporeal membrane oxygenation (ECMO) is an efficient approach in the management of refractory cardiogenic shock. The recently published SAVE-score was designed as a tool to predict in-hospital survival in patients receiving ECMO for refractory cardiogenic shock [1] .

Our objective was to externally validate the SAVE-score in a new dataset, to support its clinical application.

\section{Materials and methods}

After institutionnal approval, data from every patients receiving veno-arterial-ECMO primarily for cardiogenic shock between January 2005 and December 2014 were extracted from our local single-center prospectively-maintained cardiac surgery database. Using the original publication exclusion criteria, SAVE-score calculation was performed for each patient, omitting individuals with missing data. Discrimination was assessed using the area under the receiver operator characteristic curve (AUC). Calibration was evaluated with the Hosmer-Lemeshow goodness-of-fit test and calibration plot. Slope and intercept values were calculated using logistic regression.

\section{Results and discussion}

We included $\mathbf{3 0 3}$ patients receiving ECMO. Using only complete data, SAVE-score calculation was perform in 180 patients. Overall in-hospital survival in complete-case analysis was $46.7 \%$ and did not differ from missing-data group (43,9 \%, $p=0.63)$. The SAVE-score discriminated survivors and non survivors with an AUC of $0.746(95 \% \mathrm{Cl} 0.674$ to $0.817, p=0.05)$, demonstrating good discriminatory performance. $P$ value from Hosmer-Lemeshow goodness-of-fit test was 0.98 indicating no evidence of poor calibration. However, calibration plot analysis (Fig. 1) revealed both underfitting (calibration slope $=1,22$ ) and overestimation (intercept $\mathbf{= 0 , 1 4}$ ) resulting in predicted survival rates not varying enough and being systematically too high.

SAVE-score items ${ }^{1}$
Acute cardiogenic shock diagnosis group
Age
Weight
Acute pre-ECMO organ failures
Chronic renal failure
Duration of intubation prior to initiation of ECMO
Peak inspiratory pressure $\leq 20 \mathrm{cmH} 2 \mathrm{O}$
Pre-ECMO cardiac arrest
Diastolic blood pressure before $\mathrm{ECMO} \geq 40 \mathrm{mmHg}$
Pulse pressure before ECMO $\leq 20 \mathrm{mmHg}$
HCO3 before ECMO $\leq 15 \mathrm{mmol} / \mathrm{L}$

\section{Conclusion}

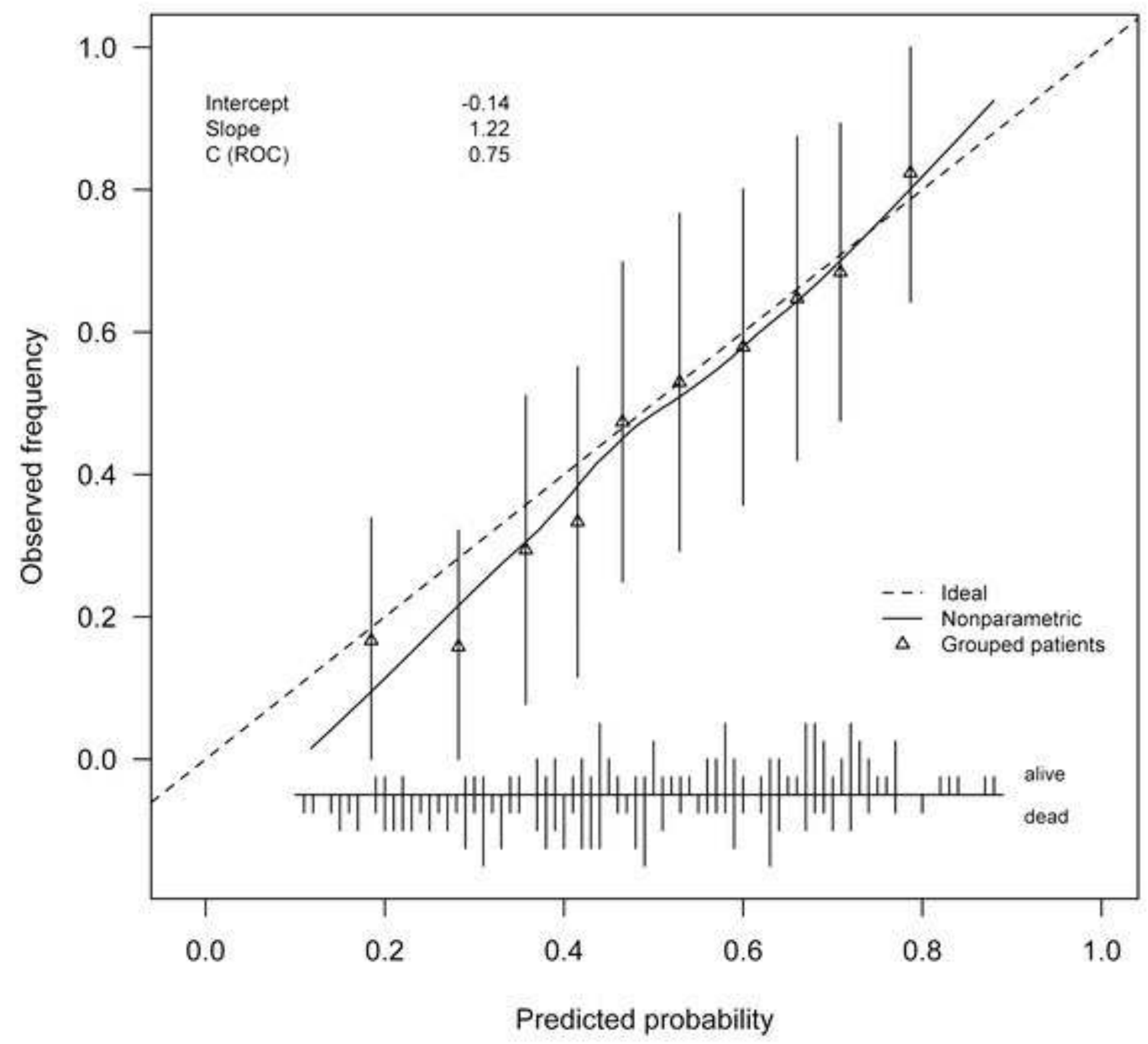

External validation of the SAVE-score in our independant cohort revealed good discriminatory performance and acceptable calibration. However, model updating by recalibration might be necessary before clinical application. 